\title{
Total Parenteral Nutrition Management Based on Intravenous Drug Compounding Service
}

\author{
Lin Lin $^{1}$, Fasheng Luo ${ }^{1}$, Amiya Bhaumik ${ }^{1} \&$ Divya Midhun Chakkaravarthy $^{1}$ \\ ${ }^{1}$ Department of Health Sciences, Lincoln University College, Selangor Darul Ehsan, Malaysia \\ Correspondence: Lin Lin, Department of Health Sciences, Lincoln University College, Selangor Darul Ehsan, \\ Malaysia. Tel: 86-152-2155-3469.
}

Received: October 27, 2020 Accepted: November 30, 2020 Online Published: January 3, 2021

doi:10.5539/gjhs.v13n2p84 URL: https://doi.org/10.5539/gjhs.v13n2p84

\begin{abstract}
Total parenteral nutrition treatment is complex and has serious and detrimental complications, including catheter-related blood stream infections, fluid and electrolyte imbalance, hyperglycemia and hypercalcemia. This research examines how Intravenous Drug Compounding Service (IDCS) figures in total parenteral nutrition prescriptions reviewed by pharmacists, strengthens hospital clinical care and improves patients' health. For this study, a total of 56164 nutritional prescriptions or medical orders from a hospital in Guangdong from 2016 to 2020 were randomly selected. According to whether IDCS was administered, the patients were divided into two experimental groups: the intervention group and the control group. The types and numbers of irrational prescriptions in the two groups were analyzed and compared, and further analysis of the role of IDCS in improving the level of nutritional prescriptions was performed. The results showed that the rate of irrational prescriptions in the intervention group from 2016 to 2020 was significantly lower than that in the control group, and the difference was statistically significant $(\mathrm{P}<0.01)$. The total irrational prescription rate in the intervention group was 32.53\%o on average, which was significantly lower than the $156 \%$ in the control group. The difference was statistically significant $(\mathrm{P}<0.01)$. After the administration of IDCS, the incidence rates of both total prescription errors and formulation errors were significantly lower than the incidence rate of irrational prescriptions without pharmacists' intervention. The study confirms the importance and necessity of IDCS, so that patients can receive more efficient nutrition and health management services.
\end{abstract}

Keywords: total parenteral nutrition, Intravenous Drug Compounding, health management

\section{Introduction}

Intravenous administration, including intravenous drip and intravenous infusion, is a commonly used method of clinical practice. Intravenous infusion can adjust the body's water percentage, salt and electrolyte imbalance, and supplement nutrients. However, during intravenous infusion, nutrients or drugs enter the human blood circulatory system directly through the vein, and any improper drug configuration before medication or any improper medication process can cause serious adverse reactions, even death, in patients. Total Parenteral Nutrition (TPN) is a technology that provides all the nutrition the patient needs through a vein, bypassing the digestive tract. It is mainly used for patients who cannot absorb nutrients via the digestive tract, like those receiving high-dose chemotherapy, radiotherapy and bone marrow transplantation, those suffering from malnutrition caused by gastrointestinal dysfunctions, moderate or severe acute pancreatitis, and patients with the severe metabolic syndrome.

Total parenteral nutrition treatment has become one of the most important medical treatments helping maintain life. Total parenteral nutrition is a complex treatment method. When the patient cannot get enough water and nutrients through the oral or intestinal route, the nutrition (glucose, amino acid, lipids, electrolytes, vitamins and trace minerals) enters the body intravenously (Dudrick \& Palesty, 2011; Koss \& Joshi, 2003; Vinnars \& Wilmore, 2003). The proportion of intravenous drug administration in China is high, and drug compatibility is generally determined by nursing staff on duty in an open ward environment (Lifang et al., 2018). In recent years, due to the rapid development of new drugs and dosage forms, a large number of new domestic and foreign drugs have entered the market, making the decisions about compatibility of different drugs more and more complicated, and increasing the incidence rate of drug compatibility errors. Therefore it is difficult to ensure the compatibility of drugs, especially during the configuration of cytotoxic drugs. This has increased the difficulty and risk of clinical 
medication, and at the same time posing a certain hazard to the health of nursing staff and the hospital environment.

Intravenous drug compounding service (IDCS) complies with the production quality management specifications of drugs. To provide intravenous therapy, specially trained pharmacy technicians strictly follow the standard operating procedures reviewed by pharmacists. For rational drug use and to provide clinical nutritional supplements, drugs and other prescriptions are configured for intravenous administration. IDCS is to strengthen clinical nursing work in hospitals and provide high-quality nursing services to patients. It is an important tool for deepening the reform of the medical and health system and implementing new scientific concepts (Jia, Fan, $\mathrm{Hu}$, Zhao \& Sun, 2018). In the 1960s, many medical institutions in the United States repeatedly reported medical accidents that had occurred due to errors in the mixing or configuration of intravenous infusions, such as presence of drugs (medicine previously administered by doctors) in blood or drug contamination during intravenous infusion configuration, causing heat source reactions in patients. IDCS in China began relatively late. The Jingan District Central Hospital in Shanghai established the first IDCS in 1999, followed by Beijing, Guangdong, Shandong, Jiangsu and other regions. More and more medical institutions in China are introducing IDCS, which plays an irreplaceable role in reducing drug waste, avoiding cross-contamination, promoting rational drug use, and development of hospital services. The establishment of IDCS can make intravenous nutrition treatment more effective. It is a new research topic and one of innovations in modern health care (Yan, Xing-Ru, \& Yi, 2019; Shuying, 2014).

In an open working environment, while relevant training and occupational protection of nursing personnel is relatively poor, prescriptions for total parenteral nutrition, antibiotics, and cytotoxic drugs cannot guarantee the quality of infusions. In addition, there is a big difference between pharmacy work and nursing work. When nurses work alone, it is difficult to ensure the accuracy of intravenous drug dispensing operation, and the quality of infusion drugs cannot be guaranteed. Therefore, it is particularly important to implement centralized allocation of prescriptions for antibiotics, cytotoxic drugs and total parenteral nutrition drugs. In order to standardize drug management, reduce medication errors, promote clinical rational use of drugs, and improve the quality of health management, the construction of IDCS is imperative. IDCS can standardize drug management, reduce medication errors, promote clinical rational drug use, and improve nursing quality. It can also strengthen occupational protection of medical staff, reduce medical costs and improve clinical medical services (Xue, Tang, \& Lian, 2003; Jing \& Ping, 2019).

\section{Method}

IDCS is based on the characteristics of nutrients or drugs, and in a specific operating environment, specially trained pharmaceutical technicians strictly follow the standard operating procedures. After reasonable allocation of prescriptions for total intravenous nutrition drugs, antibiotic drugs and cytotoxic drugs that have been reviewed by pharmacists, pharmaceutical services are provided for clinical rational use of drugs and drug treatment. This study explores the organizational structure and full set of management models of a newly-built IDCS in Zhonde hospital in China and examines the role of IDCS in improving the level of nutrition prescriptions, achieving improved drug management and application models, strengthening hospital infrastructure construction, and enhancing nutrition management services.

This study randomly selected 56164 prescriptions or medical orders from Zhonde hospital in China during the five years from 2016 to 2020. All patients' personal information and disease conditions have been kept confidential, and all prescriptions or medical orders were classified according to whether the IDCS pharmacist took corresponding intervention measures. For the intervention group, the IDCS pharmacist regularly communicated with the doctors in the wards of each department, analyzed and summarized the problems and unreasonable use of drugs by the doctors in the wards, and provided feedback to the doctors. The prescriptions of the control group were prepared by IDCS, and the pharmacist did not take corresponding intervention measures. The number, types, and complaints about irrational prescriptions in the two groups were analyzed and compared. The numbers of irrational prescriptions were counted separately for intervention group and control group, taking into account whether IDCS pharmacists took appropriate intervention measures. Then it was further analyzed how IDCS improved the efficiency of prescriptions.

In order to summarize and analyze the irrational prescriptions reviewed, the review of prescriptions by pharmacists was mainly based on the drug inserts, the Pharmacopoeia of the People's Republic of China and the Instructions for Clinical Use, etc. The SPSS 20.0 statistical software was used to analyze the data, counting the number of questionable doctor orders in both groups and calculating their rate, which is the number of questionable doctor orders divided by total number of doctor orders times $100 \%$. The types of irrational prescriptions, the proportion of 
each type in all irrational prescriptions, the proportion of irrational prescriptions of a certain type (which equals the number of irrational prescriptions of this type divided by the number of irrational prescriptions times $100 \%$ ) were counted and analyzed. To compare whether there was a difference between the two groups of questionable doctor orders rate and the proportion of irrational prescription types between the two groups, the $\mathrm{X}^{2}$ test was used, and the difference amounted to $\mathrm{P}<0.05$, which was statistically significant.

\section{Results \& Discussion}

The numbers of random samples of prescriptions or medical orders for each year from 2016 to 2020 were respectively $10134,10458,10653,11853$ and 13066. Out of 56164 prescriptions or medical orders in 5 years, 360 were irrational prescriptions, accounting for $64.10 \%$ of the total. The number of irrational prescriptions in each year was respectively $63,71,75,67$, and 84 , and the irrational prescription rates were respectively $62.17 \%$ o, 67.89 $\%$ o, $70.40 \%$ oo, $56.53 \%$, and $64.29 \%$ o. (See Table 1.)

Table 1. Number of irrational prescriptions in Zhonde hospital during 2016 to 2020

\begin{tabular}{llllllc}
\hline & 2016 & 2017 & 2018 & 2019 & 2020 & 5-year total \\
\hline The total number of prescriptions or orders & 10134 & 10458 & 10653 & 11853 & 13066 & 56164 \\
Number of irrational prescriptions & 63 & 71 & 75 & 67 & 84 & 360 \\
irrational prescription rate \%o & 62.17 & 67.89 & 70.40 & 56.53 & 64.29 & 64.10 \\
\hline
\end{tabular}

According to the statistics on irrational prescriptions of different types of drugs in the hospital in the past five years, it was found that there were irrational prescriptions in the prescriptions of total intravenous nutrition drugs, antibiotic drugs, cytotoxic drugs and common drugs. The numbers of irrational prescriptions in total intravenous nutrition, antibiotics, cytotoxic drugs and general drug prescriptions from 2016 to 2020 were respectively: 83, 108, 114 , and 55. The cumulative number of irrational prescriptions in 5 years was 360 ; the proportions were respectively $23.06 \%, 30.00 \%, 31.67 \%, 15.28 \%$. In 2016 and 2019, the highest proportion of irrational prescription types were total intravenous nutrition drugs, accounting for $41.27 \%$ and $31.34 \%$ respectively. (See Table 2 and Table 3.)

Table 2. Number of irrational prescriptions for different types of drugs in Zhonde hospital from 2016 to 2020

\begin{tabular}{|c|c|c|c|c|c|c|}
\hline Types of drugs & 2016 & 2017 & 2018 & 2019 & 2020 & total \\
\hline Antibiotic drugs & 15 & 16 & 16 & 17 & 19 & 83 \\
\hline Cytotoxic drugs & 15 & 24 & 24 & 19 & 26 & 108 \\
\hline Total parenteral nutrition & 26 & 20 & 22 & 22 & 25 & 114 \\
\hline Common drugs & 7 & 11 & 13 & 10 & 14 & 55 \\
\hline Total & 63 & 71 & 75 & 67 & 84 & 360 \\
\hline
\end{tabular}

Table 3. Proportion of irrational prescription types in Zhonde hospital from 2016 to 2020 (\%)

\begin{tabular}{lllllll}
\hline Types of drugs & 2016 & 2017 & 2018 & 2019 & 2020 & total \\
\hline Antibiotic drugs & 23.81 & 22.54 & 21.33 & 25.37 & 22.62 & 23.06 \\
Cytotoxic drugs & 23.81 & 33.80 & 32.00 & 28.36 & 30.95 & 30.00 \\
Total parenteral nutrition & 41.27 & 28.17 & 29.33 & 31.34 & 29.76 & 31.67 \\
Common drugs & 11.11 & 15.49 & 17.33 & 14.93 & 16.67 & 15.28 \\
Total & 100 & 100 & 100 & 100 & 100 & 100 \\
\hline
\end{tabular}

All extracted prescriptions or medication orders were divided into intervention group and control group according to whether the pharmacist took corresponding intervention measures. In the intervention group, the IDCS pharmacist regularly communicated with doctors in each department's ward, analyzed and summarized the problems and unreasonable use of drugs by the ward doctors, and provided feedback to the clinical doctors in 
relevant departments. The prescription of the control group was prepared by IDCS, and the pharmacist did not take corresponding intervention measures. After the classification, the total number of prescriptions or doctor's orders in the intervention group was 41805 , the total number of prescriptions or doctor's orders in the control group was 14359; the intervention group received 136 irrational prescriptions, and the control group received 227 irrational prescriptions. The types of prescription errors were divided into four categories: medical order errors, dispensing errors, medication errors, and other errors. The four types of errors and their numbers in the two groups from 2016 to 2020 are shown in Tables 4 and 5.

From 2016 to 2020, the total number of prescriptions or doctor orders in the intervention group were 7727, 7954, 8011,8547 , and 9566 , respectively, and the 5-year total was 41805 . The total number of prescriptions or doctor orders in the control group were 2407, 2504, 2642, 3306, and 3500, respectively, and the 5-year total was 14359. During 2016 to 2020, the incidence rate of irrational prescriptions in the intervention group was $28.47 \%$ o, 36.46 $\%$ o, $33.70 \%$ oo, $30.4 \%$ oo, $233.45 \%$, and the total incidence rate of irrational prescriptions was $32.53 \%$. During the past five years, the control group was prescribed unreasonable $170.34 \%$ o, $167.73 .6 \%$ oo, $181.68 \%$, 124.02 $\%$, $148.57 \%$; the total of unreasonable formulations was $156.00 \%$. The rate of irrational prescriptions in the intervention group was significantly lower than that in the control group, and the difference was statistically significant $(\mathrm{P}<0.01)$. The total irrational prescription rate in the intervention group was $32.53 \%$ on average, which was significantly lower than the $156.00 \%$ in the control group. The difference was statistically significant $(\mathrm{P}<0.01)$.

Table 4. Comparison of the number of irrational prescriptions between the two groups in Zhonde hospital from 2016 to 2020

\begin{tabular}{lllllll}
\hline Group & time & Medical order error & Allocation error & Administration error & Other errors & total \\
\hline \multirow{5}{*}{ Intervention group } & 2016 & 1 & 13 & 6 & 2 & 22 \\
& 2017 & 2 & 18 & 5 & 4 & 29 \\
& 2018 & 1 & 15 & 8 & 3 & 27 \\
& 2019 & 1 & 17 & 6 & 2 & 26 \\
& 2020 & 2 & 20 & 7 & 3 & 32 \\
& total & 7 & 83 & 32 & 14 & 136 \\
\hline \multirow{5}{*}{ Control group } & 2016 & 2 & 26 & 8 & 5 & 41 \\
& 2017 & 2 & 23 & 12 & 5 & 42 \\
& 2018 & 3 & 26 & 13 & 6 & 48 \\
& 2019 & 2 & 24 & 10 & 5 & 41 \\
& 2020 & 3 & 30 & 12 & 7 & 52 \\
& total & 12 & 129 & 55 & 28 & 224 \\
\hline
\end{tabular}

Table 5. Comparison of irrational prescription rates between the two groups in Zhonde hospital from 2016 to 2020 (numbers and \%oo)

\begin{tabular}{llllllll}
\hline Group & & 2016 & 2017 & 2018 & 2019 & 2020 & total \\
\hline \multirow{4}{*}{ Intervention group } & The total number of prescriptions or orders & 7727 & 7954 & 8011 & 8547 & 9566 & 41805 \\
& Number of irrational prescriptions & 22 & 29 & 27 & 26 & 32 & 136 \\
& irrational prescription rate (\%oo ) & 28.47 & 36.46 & 33.70 & 30.42 & 33.45 & 32.53 \\
\hline \multirow{3}{*}{ Control group } & The total number of prescriptions or orders & 2407 & 2504 & 2642 & 3306 & 3500 & 14359 \\
& Number of irrational prescriptions & 41 & 42 & 48 & 41 & 52 & 224 \\
& irrational prescription rate (\%oo ) & 170.34 & 167.73 & 181.68 & 124.02 & 148.57 & 156.00 \\
\hline $\mathrm{X}^{2}$ & & 59.787 & 48.670 & 62.232 & 37.156 & 53.164 & 255.829 \\
\hline $\mathrm{P}$ & & $<0.001$ & $<0.001$ & $<0.001$ & $<0.001$ & $<0.001$ & $<0.001$ \\
\hline
\end{tabular}


Based on the above investigation, the following results were obtained:

(1) Irrational prescriptions. There were 360 irrational prescriptions in the 5614 prescriptions or doctor's orders, accounting for $64.10 \%$; the irrational prescription types from 2016 to 2020 included overdose prescriptions, improper solvent usage, contraindications, improper choice of solvents, incorrect doctor orders, and incorrect drug usage. There were seven types of dosing frequency errors. The most irrational prescription types from 2016 to 2020 were all improper solvent usage; antibiotic drugs, cytotoxic drugs, total parenteral nutrition drugs and general drug prescriptions all had irrational prescriptions. The highest proportion of irrational prescription types in 2016 and 2019 was for total parenteral nutrition drugs, accounting for $41.27 \%$ and $31.34 \%$ respectively; the highest proportion of irrational prescription types in 2017, 2018 and 2020 were all cytotoxic drugs. They accounted for $33.80 \%, 32.00 \%$ and $30.95 \%$ respectively.

(2) The numbers of irrational prescriptions and types of irrational prescriptions in the second group: from 2016 to 2020 there were 41805 prescriptions or doctor orders in the intervention group, as compared to 14359 in the control group. There were 136 irrational prescriptions in the intervention group, accounting for $32.53 \%$, and 227 irrational prescriptions in the control group, accounting for $156.00 \%$. The rate of irrational prescriptions in the intervention group from 2016 to 2020 was significantly lower than that of the control group, and the difference is statistically significant $(\mathrm{P}<0.01)$; the total irrational prescription rate in the intervention group from 2016 to 2020 averaged 32.53\%oo, which was significantly lower than in the control group. The difference between the rates of the two groups is statistically significant $(\mathrm{P}<0.01)$. The incidence rate of medical order errors in the intervention group in 2018 was significantly lower than that of the control group, and the difference is statistically significant $(\mathrm{P}<0.01)$. The total medical order error incidence rate in the intervention group from 2016 to 2020 was $1.67 \%$, significantly lower than in the control group's $8.36 \%$; the difference is statistically significant $(\mathrm{P}<0.01)$. The incidence rate of deployment errors in the intervention group in 2016-20120 was significantly lower than in the control group, and the difference is statistically significant $(\mathrm{P}<0.01)$. The total incidence rate of deployment errors in the intervention group from 2016 to 2020 was $19.85 \%$ oo significantly lower than in the control group (89.84 \%oo), and the difference is statistically significant $(\mathrm{P}<0.01)$. The incidence rate of medication errors in the intervention group from 2016 to 2020 was significantly lower than that in the control group, and the difference is statistically significant $(\mathrm{P}<0.01)$. The total incidence rate of medication errors in the intervention group from 2016 to 2020 was $7.65 \%$, significantly lower than the control group's $38.30 \%$; the difference is statistically significant $(\mathrm{P}<0.01)$. The incidence rate of other errors in the intervention group from 2016 to 2020 was significantly lower than in the control group, and the difference is statistically significant $(\mathrm{P}<0.01)$. The total incidence rate of other errors in the intervention group from 2016 to 2020 was 3.35\%oo significantly lower than the control group's $19.50 \%$; the difference is statistically significant $(\mathrm{P}<0.01)$.

Among the common irrational prescriptions, the improper dosage of solvents is more prominent in the configuration of intravenous drugs, therefore it is especially necessary to pay attention to it in future work. Irrational prescriptions of total parenteral nutrition drugs and cytotoxic drugs are the most common. IDCS pharmacists should strengthen their intervention in the administration of total parenteral nutrition drugs and cytotoxic drugs, striving to reduce the proportion of irrational prescriptions of total intravenous drugs and cytotoxic drugs. After the intervention of IDCS pharmacists, the incidence rate of total prescriptions or medical order errors drops well below the incidence rate of irrational prescriptions without pharmacists' intervention. It reduced the incidence rate of complaints about intravenous medication, improved the quality of care, and enabled patients to receive better and more reasonable clinical health and medical management services. This indicates that after the establishment of IDCS the intervention of pharmacists has obviously improved the situation, which confirms the importance and need for pharmacists in IDCS.

\section{Conclusion}

There were irrational prescriptions in all four categories of prescriptions of total parenteral nutrition drugs, antibiotic drugs, cytotoxic drugs and common drugs. Irrational prescriptions of total parenteral nutrition drugs were the most common. We suggest that IDCS pharmacists strengthen their intervention in the administration of total parenteral nutrition drugs and strive to reduce the unreasonable proportion of total parenteral nutrition prescriptions. After the intervention of IDCS pharmacists, the total incidence rates of irrational prescriptions, including the incidence rates of medical order errors, deployment errors, medication errors, and other errors became significantly lower than the incidence rate of irrational prescriptions without pharmacists' intervention. The research results show the importance and need for pharmacists in IDCS. After the establishment of IDCS, the intervention of pharmacists significantly reduces the incidence rate of complaints about intravenous medication, improves prescription standards and the quality of care, and enables patients to receive more scientific and 
reasonable nutrition and health services.

After studying a large amount of relevant data, we have comprehensively analyzed the structure and working mode of IDCS, discussed the necessity and importance of establishing IDCS, and finally elaborated on the scale of personnel requirements and full set of management modes of the hospitals to be equipped with IDCS. In our analysis of the role of IDCS in improving the level of prescriptions, the nutrition prescriptions of a hospital in Guangdong from 2016 to 2020 were selected for analysis, and the IDCS pharmacists were divided into two experimental groups according to whether the IDCS pharmacists took corresponding intervention measures. The number and types of irrational prescriptions in the second group were analyzed statistically, and finally the role of IDCS in improving prescription standards and the importance of pharmacist intervention was stressed. This research is of significant practical value for improving total parenteral nutrition management.

\section{Competing Interests Statement}

The authors declare that there are no competing or potential conflicts of interest.

\section{References}

Along, K., Jing, Z., \& Fengxia, G. (2015). Risk assessment of safe operation in the intravenous drug compounding service and the establishment of the prevention system. Pharmaceutical Care and Research.

Chen, C., Sheng, L., Weiping, X., Hong, X., \& Hospital, A. P. (2013). Analysis on irrational medication orders of intravenous drug compounding service in our hospital. China Pharmaceuticals.

Dudrick, S. J., \& Palesty, J. A. (2011). Historical highlights of the development of total parenteral nutrition. Surgical Clinics of North America, 91, 693-717. https://doi.org/10.1016/j.suc.2011.02.009

Fu, J.-Y. (2003). Application of intravenous drug compounding service has been applied in clinic. Journal of Nursing Administration.

Jia, X., Fan, J., Hu, X., Zhao, S., \& Sun, W. (2018). Causes of drug breakage in intravenous drug compounding service and intervention measures. Pharmaceutical Care and Research, 18(3), 218-220.

Koss, W., \& Joshi, N. (2003). Prescribing total parenteral nutrition. Nutrition, 19(9), 819-820. https://doi.org/10.1016/S0899-9007(03)00102-3

Lifang, Z., Zhihua, L., Yali, D., Chunmei, L., Yanchun, Z., \& Jiangmei, Q. (2018). Rational drug use in primary care in highly focused areas during the comprehensive reform of primary care system in china. Chinese General Practice.

Liu, R., Ding, S. H., \& Dong, P. (2012). Exploration and practice of humanistic management in intravenous drug compounding service LJ]. Pharmaceutical Care \& Research, 12(6), 473-475. https://doi.org/10.5428/pcar20120623

Mei-Xin, N., Min, Y., Hai-Juan, G., Pharmacy, D., \& Hospital, N. T. (2013). Analysis of intervention effectiveness on non-rational use of antineoplastic agents in intravenous drug compounding service (IDCS). Evaluation and Analysis of Drug-Use in Hospitals of China.

Paoletti, R. D. (2000). Casey E W. Reducing costs through centralization and standardization of an iv admixture program. American journal of health-system pharmacy, 57(12), 1147-1149. https://doi.org/10.1093/ajhp/57.12.1147

Peng-Fei, BI, Jing, F., Xiu-Ling, J., Shan-Shan, G., Xiao-Ying, L., \& Ping, L., et al. (2019). The establishment and practice of digital quality management system of intravenous drug compounding service. Chinese Journal of Hospital Pharmacy.

Plumridge, R. J., \& Maher, M. (1993). Justification of a pharmacy intravenous admixture service in an Australian hospital. American Journal of Hospital Pharmacy, 50(3), 463-466. https://doi.org/10.1093/ajhp/50.3.463

Ruo-Mei, X., Xi-Hua, G., Yan, L., \& Feng-Fang, X. (2015). Analysis of irrational antitumor prescriptions in intravenous drug compounding service. Evaluation and Analysis of Drug-Use in Hospitals of China.

Shuying, H. (2014). Utility of continuous quality improvement for quality management in intravenous drug compounding service. China Pharmaceuticals, 115(3), 165-168.

Tallett, E. R. (1978). Centralized intravenous admixture program for nine British hospitals. American Journal of Hospital Pharmacy, 35(2), 163-165. https://doi.org/10.1093/ajhp/35.2.163 
Vinnars, E., \& Wilmore, D. (2003). History of parenteral nutrition. Journal of Parenteral and Enteral Nutrition, 27(3), 225-231. https://doi.org/10.1177/0148607103027003225

Wei, Y., Xiu-Ying, C., \& Hai-Yan, L. (2009). The development and practice of intravenous drug compounding service. China Practical Medicine.

Xue, P., Tang, W., \& Lian, C. (2003). Development of pharmacist's role in intravenous drug compounding service. China Pharmacist.

Yan, LI, Xing-Ru, D., Wei, W., Wen-Liu, YU, Guo-Rong, S., \& Yi, LI (2019). The application of two-way precise infusion solution dispenser in intravenous drug compounding service of our hospital. Chinese Journal of Hospital Pharmacy.

Zhen, C., Quan, S. C., Yang, Z., Wi, et al. (2007). Drug administration of intravenous drug compounding service in Changhai Hospital affiliated to Second Military Medical University. Pharmaceutical Care \& Research, 2007, 7(3), 219-221.

\section{Copyrights}

Copyright for this article is retained by the author(s), with first publication rights granted to the journal.

This is an open-access article distributed under the terms and conditions of the Creative Commons Attribution license (http://creativecommons.org/licenses/by/4.0/). 